\title{
Comparison principles for fractional differential equations with the Caputo derivatives
}

\author{
Ziqiang $\mathrm{Lu}^{1}$ and Yuanguo Zhu ${ }^{1 *}$ (D)
}

"Correspondence:

ygzhu@njust.edu.cn

${ }^{1}$ School of Science, Nanjing

University of Science and

Technology, Nanjing, China
The Author(s) 2018. This article is distributed under the terms of the Creative Commons Attribution 4.0 International License (http://creativecommons.org/licenses/by/4.0/), which permits unrestricted use, distribution, and reproduction in any medium, provided you give appropriate credit to the original author(s) and the source, provide a link to the Creative Commons license, and indicate if changes were made.

\begin{abstract}
In this paper, we deal with comparison principles for fractional differential equations involving the Caputo derivatives of order $p$ with $0 \leq n-1<p \leq n$. First, we present comparison results with strict inequalities for fractional differential equations with the Caputo derivatives. Then we investigate local existence and extremal solutions for fractional differential equations with the Caputo derivatives. Finally, we consider comparison results with nonstrict inequalities for fractional differential equations with the Caputo derivatives.
\end{abstract}

MSC: Primary 26A33; 26D10; secondary 34A12; 34A40

Keywords: Comparison principle; Fractional differential equation; Caputo derivatives; Local existence; Extremal solutions

\section{Introduction}

The theory of fractional differential equations with the Caputo derivatives greatly attracted the attention of many scholars for their wide applications to various fields, such as in physics, engineering, electrochemistry, and so on; see [1-3] and related references therein. The mathematical modeling and simulation of systems and processes based on the fractional derivatives of the Caputo type describing their properties naturally lead to the necessity to solve these fractional differential equations. Analytic solutions are usually provided for some special fractional differential equations with the Caputo derivatives. There is a growing interest in the existence theory and properties of solutions and numerical treatments for fractional differential equations. The comparison principle plays an important role in the study of the numerical solutions and properties of fractional differential equations.

Many researchers studied the fractional differential equations involving the RiemannLiouville derivatives of order $p(0<p<1)$. Hadid et al. [4] presented the local existence and uniqueness of solutions of nonlinear differential equations. Lakshmikantham and Vatsala [5-7] investigated some quantitative properties for fractional differential equations with the Riemann-Liouville derivatives by employing differential and integral inequalities. They investigated the comparison theorem for Riemann-Liouville-type fractional 
differential inequalities $(0<p<1)$ of the form

$$
D^{p} v(t) \leq f(t, v(t)), \quad D^{p} w(t) \geq f(t, w(t)), \quad 0<t \leq T,
$$

where $D^{p} v(t)$ represents the Riemann-Liouville derivative of $v(t)$ of order $p$. When one of the inequalities is strict, we have $v(t)<w(t)$ if $v(0)<w(0)$. If both inequalities are nonstrict, then $v(t) \leq w(t)$ if $v(0) \leq w(0)$ and $L T^{p} \leq \frac{1}{\Gamma(1-p)}$, where $L$ is a Lipschitz constant satisfying $|f(t, x)-f(t, y)| \leq L|x-y|$. Denton and Vatsala [8] further developed these properties for fractional integral equations of the form

$$
\begin{array}{ll}
w(t) \geq \frac{w_{a}}{\Gamma(p)}(t-a)^{p-1}+D^{-p} f(t, w), & a<t \leq T, \\
v(t) \leq \frac{v_{a}}{\Gamma(p)}(t-a)^{p-1}+D^{-p} f(t, v), & a<t \leq T,
\end{array}
$$

where $w_{a}=\left.\Gamma(q) w(t)(t-a)^{1-p}\right|_{t=a}$ and $v_{a}=\left.\Gamma(q) v(t)(t-a)^{1-p}\right|_{t=a}$. Then if one of inequalities is strict, then $v_{a}<w_{a}$ implies $v(t)<w(t)$. The other conclusion $v(t) \leq w(t)$ holds if $v_{a} \leq w_{a}$ and $f(t, x)$ satisfies the right-sided Lipschitz condition. Meanwhile, they presented the Gronwall inequalities for the Riemann-Liouville-type fractional differential equations. Many other researches can be seen in [9-16] and related references therein.

However, for the Riemann-Liouville case, one would have to specify the values of certain fractional derivatives (and integrals) of the unknown solution at the initial point. The Riemann-Liouville derivative is more commonly applied in pure mathematics. The Caputo derivative can just overcome these defects, since the initial values $x(0), x^{\prime}(0), \ldots, x^{(n-1)}(0)$ are specified and have a well-understood physical meaning. In this case, the comparison principles for Caputo-type fractional differential equations are important for the study of properties of their solutions. As we know, the work on comparison principles for fractional differential equations involving the Caputo derivative of order $p$ $(0 \leq n-1<p \leq n)$ is yet to be initiated. In this paper, we present comparison principles for fractional differential equations involving the Caputo derivatives of order $p(n-1<p \leq n)$ under strict and nonstrict inequalities and investigate the existence of extremal solutions to fractional differential equations with the Caputo derivatives.

The remainder of this paper is organized as follows. In Sect. 2, we recall two types of fractional derivatives with some properties. In Sect. 3, we present comparison results under strict inequality conditions for the fractional differential equations involving the Caputo derivatives of order $p(0 \leq n-1<p \leq n)$ with initial conditions. In Sect. 4, we investigate the local existence theorem and extremal solutions to fractional differential equations involving the Caputo derivatives with initial value conditions. In Sect. 5, we consider the comparison principles under nonstrict inequality conditions for fractional differential equations with the Caputo derivatives by employing the theorems proposed in the previous sections.

\section{Preliminaries}

In this section, we recall two types of fractional order derivatives of a function with some properties. 
Definition 1 For $p>0$ and $f \in C[a, b]$. The $p$ th fractional integral of $f(t)$ is defined by

$$
I_{a+}^{p} f(t)=\frac{1}{\Gamma(p)} \int_{a}^{t}(t-s)^{p-1} f(s) \mathrm{d} s
$$

Definition 2 Let $0 \leq n-1<p \leq n$ and $f:[a, b] \rightarrow R$ be a differentiable function of at least order $n$. Then the $p$ th Caputo fractional derivative of $f(t)$ is defined by

$$
{ }^{c} D_{a+}^{p} f(t)=\frac{1}{\Gamma(n-p)} \int_{a}^{t}(t-s)^{n-p-1} f^{(n)}(s) \mathrm{d} s,
$$

where $f^{(n)}(t)$ is the $n$ th-order derivative of $f(t)$.

Definition 3 Let $0<p<1$ and $f \in C[a, b]$. The $p$ th Riemann-Liouville fractional derivative of $f(t)$ is defined by

$$
D_{a+}^{p} f(t)=\frac{1}{\Gamma(1-p)} \frac{\mathrm{d}}{\mathrm{d} t} \int_{a}^{t}(t-s)^{-p} f(s) \mathrm{d} s
$$

Now we give some properties of the fractional integral and Caputo and RiemannLiouville fractional derivatives:

(i) These two types of fractional derivatives satisfies $(0<p<1)$ are related as follows:

$$
{ }^{c} D_{a_{+}}^{p} f(t)=D_{a_{+}}^{p} f(t)-\frac{(t-a)^{-p}}{\Gamma(1-p)} f(a) .
$$

(ii) The fractional derivative and the fractional integral are related as follows $(0 \leq n-1<$ $p \leq n)$ :

$$
{ }^{c} D_{a+}^{p} I_{a+}^{p} f(t)=f(t), \quad I_{a+}^{p}{ }^{c} D_{a+}^{p} f(t)=f(t)-\sum_{k=0}^{n-1} \frac{(t-a)^{k}}{\Gamma(k+1)} f^{(k)}(a) .
$$

(iii) Integer-order and fractional derivatives satisfy the equality $(p>0)$

$$
{ }^{c} D_{a+}^{n+p} f(t)={ }^{c} D_{a+}^{p} D^{n} f(t) .
$$

Remark 1 We abbreviate $I_{0+}^{p}, D_{0+}^{p}$, and ${ }^{c} D_{0+}^{p}$ as $I^{p}, D^{p}$, and ${ }^{c} D^{p}$, respectively.

\section{Comparison principles with strict inequalities}

Consider the following initial value problem (IVP) with Caputo-type fractional differential equation:

$$
\left\{\begin{array}{l}
{ }^{c} D^{p} x(t)=f(t, x(t)) \\
x^{(k)}(0)=x_{k}, \quad k=0,1, \ldots, n-1,
\end{array}\right.
$$

where $f \in C([0, T] \times R, R)$ and $0 \leq n-1<p \leq n$. The IVP (3) is equivalent to the following Volterra fractional integral equation:

$$
x(t)=\sum_{k=0}^{n-1} \frac{x_{k} t^{k}}{\Gamma(k+1)}+\frac{1}{\Gamma(p)} \int_{0}^{t}(t-s)^{p-1} f(s, x(s)) \mathrm{d} s .
$$


We need the following useful lemma.

Lemma 1 Let $m(t): R_{+} \rightarrow R$ be locally Lipschitz continuous such that, for $t_{1} \in(0,+\infty)$,

$$
m\left(t_{1}\right)=0 \text { and } m(t) \leq 0 \quad \text { for } 0 \leq t \leq t_{1} .
$$

Then $D^{p} m\left(t_{1}\right) \geq 0$ for $p \in(0,1)$.

The result of Lemma 1 was presented in [5] for a locally Hölder continuous function $m(t)$. However, the proof in [5] is wrong since it requires the condition that $\lambda+p-1>0$, where $\lambda$ is the Hölder constant for $m(t)$, but $\lambda+p-1>0$ is not always true for $p \in(0,1)$. If the Hölder continuity is replaced by the Lipschitz continuity, that is, $\lambda=1$, then $\lambda+p-1>$ 0 , and then the proof of Lemma 1 follows from [5].

Now, we present a comparison principle for fractional differential equation with the Caputo derivative under strict inequalities when $p \in(0,1)$.

Theorem 1 Assume that $f(t, x)$ and $F(t, x)$ are two continuous functions defined on $G=$ $[0, T] \times R$ satisfying the inequality

$$
f(t, x)<F(t, x) \quad \text { for }(t, x) \in G
$$

Suppose that $x=\varphi(t)$ and $x=\phi(t)$ are solutions of the following initial value problems $(0<p<1)$ :

$$
\begin{aligned}
& \left(E_{1}\right): \quad{ }^{c} D^{p} x=f(t, x), \quad x(0)=x_{0}, \\
& \left(E_{2}\right): \quad{ }^{c} D^{p} x=F(t, x), \quad x(0)=x_{0},
\end{aligned}
$$

respectively. Then we have

$$
\varphi(t)<\phi(t), \quad t \in(0, T]
$$

Proof Denote $\psi(t)=\varphi(t)-\phi(t), t \in[0, T]$. We have

$$
\begin{aligned}
& \psi(0)=\varphi(0)-\phi(0)=0, \\
& { }^{c} D^{p} \psi(0)={ }^{c} D^{p} \varphi(0)-{ }^{c} D^{p} \phi(0)=f\left(0, x_{0}\right)-F\left(0, x_{0}\right)<0 .
\end{aligned}
$$

Then ${ }^{c} D^{p} \psi(t)=D^{p} \psi(t)$, and there exists $\sigma>0$ such that $\psi(t)<0$ for $0<t<\sigma$. Suppose that inequality (6) is incorrect. Then there exists at least one $t_{1}(>0)$ such that $\psi\left(t_{1}\right)=0$. Denote

$$
\beta=\inf \{t \mid \psi(t)=0, t>0\}
$$

Then $\psi(\beta)=0$ and $\psi(t)<0$ for $0<t<\beta$.

Since ${ }^{c} D^{p} \psi(t)=f(t, x)-F(t, x), t \in[0, T]$, and $f(t, x), F(t, x)$ are continuous in $G$, it follows that $\psi^{\prime}(t)$ is a locally bounded function by Definition 2 . Therefore $\psi(t)$ is locally Lipschitz continuous in $0 \leq t \leq T$. Thus $D^{p} \psi(\beta) \geq 0$ by Lemma 1 . 
On the other hand, since $\psi(\beta)=0$, denote $\gamma=\varphi(\beta)=\phi(\beta)$. Then

$$
0 \leq D^{p} \psi(\beta)={ }^{c} D^{p} \psi(\beta)={ }^{c} D^{p} \varphi(\beta)-{ }^{c} D^{p} \phi(\beta)=f(\beta, \gamma)-F(\beta, \gamma) .
$$

It is obvious that (7) is in contradiction with (5). Hence (6) is proved.

Remark 2 When $p=1$, Theorem 1 is also true as the well-known comparison theorem for ordinary differential equations with initial value condition.

Next, we extend Theorem 1 to the case for fractional differential equations with the Caputo derivative of order $n-1<p \leq n$ with initial value conditions by employing Theorem 1.

Theorem 2 Under assumption (5), suppose that $x=\varphi(t)$ and $x=\phi(t)$ are solutions of the following initial value problems $(1 \leq n-1<p \leq n)$ :

$$
\begin{array}{ll}
\left(E_{3}\right): & { }^{c} D^{p} x=f(t, x), \quad x^{(k)}(0)=x_{k}, k=0,1,2, \ldots n-1, \\
\left(E_{4}\right): & { }^{c} D^{p} x=F(t, x), \quad x^{(k)}(0)=x_{k}, k=0,1,2, \ldots n-1,
\end{array}
$$

respectively. Then $\varphi(t)<\phi(t)$ for $t \in(0, T]$.

Proof Consider ${ }^{c} D^{p} x(t)={ }^{c} D^{q} D^{n-1} x(t)$ with $q=p-n+1 \in(0,1]$. Denote $y(t)=D^{n-1} x(t)$, $t \in[0, T]$. Then the IVPs $\left(E_{3}\right)$ and $\left(E_{4}\right)$ can be transformed into initial value problems of order $0<q \leq 1$. Indeed, we have

$$
\begin{aligned}
& { }^{c} D^{p} x(t)={ }^{c} D^{q} D^{n-1} x(t)={ }^{c} D^{q} y(t), \quad y(t)=D^{n-1} x(t), \\
& x(t)=\sum_{k=0}^{n-2} \frac{x_{k} t^{k}}{\Gamma(k+1)}+\frac{1}{\Gamma(n-1)} \int_{0}^{t}(t-s)^{n-2} y(s) \mathrm{d} s .
\end{aligned}
$$

Denote

$$
\begin{aligned}
& \hat{f}(t, y(t))=f(t, x(t))=f\left(t, \sum_{k=0}^{n-2} \frac{x_{k} t^{k}}{\Gamma(k+1)}+\frac{1}{\Gamma(n-1)} \int_{0}^{t}(t-s)^{n-2} y(s) \mathrm{d} s\right) \\
& \hat{F}(t, y(t))=F(t, x(t))=F\left(t, \sum_{k=0}^{n-2} \frac{x_{k} t^{k}}{\Gamma(k+1)}+\frac{1}{\Gamma(n-1)} \int_{0}^{t}(t-s)^{n-2} y(s) \mathrm{d} s\right) .
\end{aligned}
$$

By inequality (5) we have $\hat{f}(t, y(t))<\hat{F}(t, y(t))$ for $t \in[0, T]$. The original initial value problems $\left(E_{3}\right)$ and $\left(E_{4}\right)$ can be written as

$$
\begin{aligned}
& \left(\hat{E}_{3}\right): \quad{ }^{c} D^{q} y(t)=\hat{f}(t, y(t)), \quad y(0)=D^{n-1} x(0)=x_{n-1}, \\
& \left(\hat{E}_{4}\right): \quad{ }^{c} D^{q} y(t)=\hat{F}(t, y(t)), \quad y(0)=D^{n-1} x(0)=x_{n-1} .
\end{aligned}
$$

Assume that $\hat{\varphi}(t)$ and $\hat{\phi}(t)$ are solutions of $\left(\hat{E}_{3}\right)$ and $\left(\hat{E}_{4}\right)$, respectively. Then by Theorem 1 and Remark 2 we have $\hat{\varphi}(t)<\hat{\phi}(t)$ for $t \in(0, T]$. 
Since $x(t)$ and $y(t)$ satisfy equation (9), we obtain

$$
\begin{aligned}
\varphi(t) & =\sum_{k=0}^{n-2} \frac{x_{k} t^{k}}{\Gamma(k+1)}+\frac{1}{\Gamma(n-1)} \int_{0}^{t}(t-s)^{n-2} \hat{\varphi}(s) \mathrm{d} s \\
& <\sum_{k=0}^{n-2} \frac{x_{k} t^{k}}{\Gamma(k+1)}+\frac{1}{\Gamma(n-1)} \int_{0}^{t}(t-s)^{n-2} \hat{\phi}(s) \mathrm{d} s=\phi(t),
\end{aligned}
$$

which finishes the proof.

Example 1 Assume that $1<p \leq 2$ and that $\varphi(t)$ and $\phi(t)$ are solutions to the two initial value problems

$$
\begin{aligned}
& { }^{c} D^{p} x=x, \quad x(0)=1, x^{\prime}(0)=2, \\
& { }^{c} D^{p} x=t+x, \quad x(0)=1, x^{\prime}(0)=2,
\end{aligned}
$$

respectively, where $t \geq 0$. Then we have $x<t+x$ for $t>0$, and

$$
\begin{aligned}
& \varphi(t)=E_{p, 1}\left(t^{p}\right)+2 E_{p, 2}\left(t^{p}\right), \\
& \phi(t)=E_{p, 1}\left(t^{p}\right)+2 E_{p, 2}\left(t^{p}\right)+t^{p+1} E_{p, p+2}\left(t^{p}\right),
\end{aligned}
$$

where $E_{\alpha, \beta}(z)=\sum_{k=0}^{\infty} \frac{z^{k}}{\Gamma(k \alpha+\beta)}$. We know that $\varphi(t)<\phi(t)$ for $t>0$. This is consistent with Theorem 2.

Remark 3 Some researchers $[17,18]$ attempted to give similar comparison principles for fractional differential equations with the Caputo derivatives by the method of the integral mean value theorem, which, however, does not guarantee the correctness of their proofs. Yu et al. [19] presented comparison theorems for fractional differential equations involving the Riemann-Liouville and Caputo derivatives of order $0<p<1$ with Lipschitz condition $f(t, u)-f(t, v) \leq L(u-v)$ for $u>v$.

\section{Local existence and extremal solutions}

In this section, we investigate the existence and extremal solutions for IVP (3).

Theorem 3 Assume that $f \in C\left[R_{1}, R\right]$, where

$$
R_{1}=\left\{(t, x): 0 \leq t \leq T,\left|x(t)-\sum_{k=0}^{n-1} \frac{x_{k} t^{k}}{\Gamma(k+1)}\right| \leq K\right\},
$$

and there exists $M>0$ such that $|f(t, x)| \leq M$ in $R_{1}$. Then there exists at least one solution for IVP (3) in $t \in[0, T]$.

Proof Let $\delta>0$, and let $x_{0}(t)$ be a continuous function in the interval $[-\delta, 0]$ such that $x_{0}^{(k)}(0)=x_{k}, k=0,1, \ldots, n-1,\left|x_{0}(t)-\sum_{k=0}^{n-1} \frac{x_{k} t^{k}}{\Gamma(k+1)}\right| \leq K$ for $t \in[-\delta, 0]$, and $\left|{ }^{c} D^{p} x_{0}(t)\right| \leq M$. For $0 \leq \eta \leq \delta$, we define $x_{\eta}(t)=x_{0}(t)$ in $[-\delta, 0]$ and

$$
x_{\eta}(t)=\sum_{k=0}^{n-1} \frac{x_{k} t^{k}}{\Gamma(k+1)}+\frac{1}{\Gamma(p)} \int_{0}^{t}(t-s)^{p-1} f\left(s, x_{\eta}(s-\eta)\right) \mathrm{d} s
$$


for $t \in\left[0, \alpha_{1}\right]$, where $\alpha_{1}=\min \{\alpha, \eta\}$ and $\alpha=\min \left(\left[\frac{K}{M} \Gamma(p+1)\right]^{\frac{1}{p}}, T\right)$. Then we observe that ${ }^{c} D^{p} x_{\eta}(t)$ exists and

$$
\begin{aligned}
\left|x_{\eta}(t)-\sum_{k=0}^{n-1} \frac{x_{k} t^{k}}{\Gamma(k+1)}\right| & =\frac{1}{\Gamma(p)}\left|\int_{0}^{t}(t-s)^{p-1} f\left(s, x_{\eta}(s-\eta)\right) \mathrm{d} s\right| \\
& \leq \frac{1}{\Gamma(p)} \int_{0}^{t}(t-s)^{p-1}\left|f\left(s, x_{\eta}(s-\eta)\right)\right| \mathrm{d} s \\
& =\frac{1}{\Gamma(p)} \int_{0}^{t}(t-s)^{p-1}\left|f\left(s, x_{0}(s-\eta)\right)\right| \mathrm{d} s \\
& \leq \frac{M}{\Gamma(p)} \int_{0}^{t}(t-s)^{p-1} \mathrm{~d} s=\frac{M t^{p}}{\Gamma(p+1)} \leq K,
\end{aligned}
$$

because of the choice of $\alpha_{1}$. If $\alpha_{1}<\alpha$, then we can employ (10) to extend $x_{\eta}(t)$ as a continuously fractional differentiable function on $\left[0, \alpha_{2}\right]$, where $\alpha_{2}=\min (\alpha, 2 \eta)$. Continuing this process, we can define $x_{\eta}(t)$ over $[0, \alpha]$ so that

$$
\left|x_{\eta}(t)-\sum_{k=0}^{n-1} \frac{x_{k} t^{k}}{\Gamma(k+1)}\right| \leq K
$$

for $t \in[0, \alpha]$. Furthermore, for $0 \leq t_{1}<t_{2} \leq \alpha$, we have

$$
\begin{aligned}
x_{\eta}\left(t_{1}\right) & -x_{\eta}\left(t_{2}\right) \\
= & \sum_{k=0}^{n-1} \frac{x_{k}\left(t_{1}^{k}-t_{2}^{k}\right)}{\Gamma(k+1)}+\frac{1}{\Gamma(p)} \int_{0}^{t_{1}}\left(t_{1}-s\right)^{p-1} f\left(s, x_{\eta}(s-\eta)\right) \mathrm{d} s \\
& -\frac{1}{\Gamma(p)} \int_{0}^{t_{2}}\left(t_{2}-s\right)^{p-1} f\left(s, x_{\eta}(s-\eta)\right) \mathrm{d} s \\
= & \sum_{k=0}^{n-1} \frac{x_{k}\left(t_{1}^{k}-t_{2}^{k}\right)}{\Gamma(k+1)}+\frac{1}{\Gamma(p)} \int_{0}^{t_{1}}\left[\left(t_{1}-s\right)^{p-1}-\left(t_{2}-s\right)^{p-1} f\left(s, x_{\eta}(s-\eta)\right) \mathrm{d} s\right. \\
& -\frac{1}{\Gamma(p)} \int_{t_{1}}^{t_{2}}\left(t_{2}-s\right)^{p-1} f\left(s, x_{\eta}(s-\eta)\right) \mathrm{d} s .
\end{aligned}
$$

Then there exists $M_{1}>0$ such that

$$
\begin{aligned}
& \left|x_{\eta}\left(t_{1}\right)-x_{\eta}\left(t_{2}\right)\right| \\
& \leq\left|\sum_{k=0}^{n-1} \frac{x_{k}\left(t_{1}^{k}-t_{2}^{k}\right)}{\Gamma(k+1)}\right|+\frac{M}{\Gamma(p)}\left[\int_{0}^{t_{1}}\left[\left(t_{1}-s\right)^{p-1}-\left(t_{2}-s\right)^{p-1}\right] \mathrm{d} s\right. \\
& \left.\quad+\int_{t_{1}}^{t_{2}}\left(t_{2}-s\right)^{p-1} \mathrm{~d} s\right] \\
& \leq M_{1}\left[\left(t_{2}-t_{1}\right)+\left(t_{2}^{p}-t_{1}^{p}\right)+2\left(t_{2}-t_{1}\right)^{p}\right]<\epsilon,
\end{aligned}
$$

provided that $\left|t_{2}-t_{1}\right|<\delta_{1}=\min \left\{\delta_{1}^{\prime}, \frac{\epsilon}{3 M_{1}},\left[\frac{\epsilon}{6 M_{1}}\right]^{\frac{1}{p}}\right\}$, where $\delta_{1}^{\prime}>0$ is such that $\left|t_{2}^{p}-t_{1}^{p}\right|<\frac{\epsilon}{3 M_{1}}$ for $\left|t_{2}-t_{1}\right|<\delta_{1}^{\prime}$, since $g(t)=t^{p}, t \in[0, \alpha]$, is uniformly continuous. Then we find that the 
family $\left\{x_{\eta}(t)\right\}$ consists of a series of equicontinuous and uniformly bounded functions. The Ascoli-Arzelà theorem shows the existence of a sequence $\left\{\eta_{m}\right\}$ such that $\eta_{1}>\eta_{2}>\cdots>$ $\eta_{m} \rightarrow 0$ as $m \rightarrow \infty$ and $x(t)=\lim _{m \rightarrow \infty} x_{\eta_{m}}\left(t-\eta_{m}\right)$ exists uniformly in $[0, \alpha]$. Since $f(t, x)$ is continuous, we obtain that $f\left(t, x_{\eta_{m}}\left(t-\eta_{m}\right)\right)$ tends uniformly to $f(t, x(t))$ as $m \rightarrow \infty$. Hence, term-by-term operations of (10) with $\eta=\eta_{m}$ and $\alpha_{1}=\alpha_{m}$ yield

$$
x(t)=\sum_{k=0}^{n-1} \frac{x_{k} t^{k}}{\Gamma(k+1)}+\frac{1}{\Gamma(p)} \int_{0}^{t}(t-s)^{p-1} f(s, x(s)) \mathrm{d} s
$$

for $t \in[0, \alpha]$. Thus $x(t)$ is a solution of IVP (3) for $t \in[0, \alpha]$. By the extension method (10) there exists at least one solution for IVP (3) in the interval $[0, T]$. Therefore the theorem is proved.

Employing Theorem 2 and Theorem 3 synthetically, we present the existence of extremal solutions for IVP (3).

Theorem 4 Under the assumptions of Theorem 3, there exist extremal solutions for the IVP (3) in the interval $t \in[0, T]$.

Proof Consider the fractional differential equation with initial value conditions

$$
\left(E_{m}\right): \quad{ }^{c} D^{p} x=f(t, x)+\epsilon_{m}, \quad x^{(k)}(0)=x_{k}, k=0,1, \ldots, n-1,
$$

where $\epsilon_{m}>0(m=1,2, \ldots)$, and $\epsilon_{m}$ decreases and tends to 0 as $m \rightarrow \infty$. Then by Theorem 3 there exists at least one solution $x=\varphi_{m}(t)$ for the initial value problem $\left(E_{m}\right)$ in $0 \leq t \leq T$ :

$$
\varphi_{m}(t)=\sum_{k=0}^{n-1} \frac{x_{k} t^{k}}{\Gamma(k+1)}+\frac{1}{\Gamma(p)} \int_{0}^{t}(t-s)^{p-1}\left[f\left(s, \varphi_{m}(s)\right)+\epsilon_{m}\right] \mathrm{d} s .
$$

Moreover, there exists $M_{2}>0$ such that $\varphi_{m}(t)$ satisfies

$$
\begin{aligned}
\left|\varphi_{m}(t)\right| & =\left|\sum_{k=0}^{n-1} \frac{x_{k} t^{k}}{\Gamma(k+1)}+\frac{1}{\Gamma(p)} \int_{0}^{t}(t-s)^{p-1}\left[f\left(s, \varphi_{m}(s)\right)+\epsilon_{m}\right] \mathrm{d} s\right| \\
& \leq\left|\sum_{k=0}^{n-1} \frac{x_{k} t^{k}}{\Gamma(k+1)}\right|+\frac{2 M}{\Gamma(p)} \int_{0}^{t}(t-s)^{p-1} \mathrm{~d} s \\
& =\left|\sum_{k=0}^{n-1} \frac{x_{k} t^{k}}{\Gamma(k+1)}\right|+\frac{2 M t^{p}}{\Gamma(p+1)} \leq M_{2}
\end{aligned}
$$

for $0 \leq t \leq T$. For $0 \leq t_{1}<t_{2} \leq T$, we have

$$
\begin{aligned}
\varphi_{m}\left(t_{1}\right)-\varphi_{m}\left(t_{2}\right) \\
=\sum_{k=0}^{n-1} \frac{x_{k}\left(t_{1}^{k}-t_{2}^{k}\right)}{\Gamma(k+1)}+\frac{1}{\Gamma(p)} \int_{0}^{t_{1}}\left(t_{1}-s\right)^{p-1}\left[f\left(s, \varphi_{m}(s)\right)+\epsilon_{m}\right] \mathrm{d} s \\
\quad-\frac{1}{\Gamma(p)} \int_{0}^{t_{2}}\left(t_{2}-s\right)^{p-1}\left[f\left(s, \varphi_{m}(s)\right)+\epsilon_{m}\right] \mathrm{d} s
\end{aligned}
$$




$$
\begin{aligned}
= & \sum_{k=0}^{n-1} \frac{x_{k}\left(t_{1}^{k}-t_{2}^{k}\right)}{\Gamma(k+1)} \\
& +\frac{1}{\Gamma(p)} \int_{0}^{t_{1}}\left[\left(t_{1}-s\right)^{p-1}-\left(t_{2}-s\right)^{p-1}\right]\left[f\left(s, \varphi_{m}(s)\right)+\epsilon_{m}\right] \mathrm{d} s \\
& -\frac{1}{\Gamma(p)} \int_{t_{1}}^{t_{2}}\left(t_{2}-s\right)^{p-1}\left[f\left(s, \varphi_{m}(s)\right)+\epsilon_{m}\right] \mathrm{d} s .
\end{aligned}
$$

Then there exists $M_{3}>0$ such that

$$
\begin{aligned}
& \left|\varphi_{m}\left(t_{1}\right)-\varphi_{m}\left(t_{2}\right)\right| \\
& \leq\left|\sum_{k=0}^{n-1} \frac{x_{k}\left(t_{1}^{k}-t_{2}^{k}\right)}{\Gamma(k+1)}\right|+\frac{2 M}{\Gamma(p)}\left[\int_{0}^{t_{1}}\left[\left(t_{1}-s\right)^{p-1}-\left(t_{2}-s\right)^{p-1}\right] \mathrm{d} s\right. \\
& \left.\quad+\int_{t_{1}}^{t_{2}}\left(t_{2}-s\right)^{p-1} \mathrm{~d} s\right] \\
& \leq M_{3}\left[\left(t_{2}-t_{1}\right)+\left(t_{2}^{p}-t_{1}^{p}\right)+2\left(t_{2}-t_{1}\right)^{p}\right]<\epsilon,
\end{aligned}
$$

provided that $\left|t_{2}-t_{1}\right|<\delta_{2}=\min \left\{\delta_{2}^{\prime}, \frac{1}{3 M_{3}},\left[\frac{\epsilon}{6 M_{3}}\right]^{\frac{1}{p}}\right\}$, where $\delta_{2}^{\prime}>0$ is such that $\left|t_{2}^{p}-t_{1}^{p}\right|<\frac{\epsilon}{3 M_{2}}$ for $\left|t_{2}-t_{1}\right| \leq \delta_{2}^{\prime}$, since $g(t)=t^{p}, t \in[0, \alpha]$, is uniformly continuous. Thus the sequence $\left\{\varphi_{m}(t)\right\}$ consists of equicontinuous and uniformly bounded functions on the interval $0 \leq t \leq T$. Then by the Ascoli-Arzelà theorem the sequence $\left\{\varphi_{m}(t)\right\}$ has a uniformly convergent subsequence $\left\{\varphi_{m_{k}}(t)\right\}$. Then denote

$$
\Phi(t)=\lim _{k \rightarrow \infty} \varphi_{m_{k}}(t)
$$

Therefore $\Phi(t)$ is a solution of IVP (3).

On the other hand, since $\epsilon_{m}>0$ and $f(t, x(t))<f(t, x(t))+\epsilon_{m}, \Phi(t)$ is the maximal solution of IVP (3) by Theorem 2. In a similar way, just replacing $\epsilon_{m}$ with $-\epsilon_{m}$, we observe that there exists a minimal solution of IVP (3). Therefore, the proof is completed.

\section{Comparison principles with nonstrict inequalities}

In this section, we present comparison principles with nonstrict inequality condition for fractional differential equations with the Caputo derivatives of order $p$ with $0 \leq n-1<$ $p \leq n$.

Theorem 5 Assume that $f(t, x)$ and $F(t, x)$ are two continuous functions defined on $G=$ $[0, T] \times R$ and satisfying the inequality

$$
f(t, x) \leq F(t, x) \quad \text { for }(t, x) \in G
$$

Suppose that $x=\phi(t), \phi_{m}(t)$, and $\phi_{M}(t)$ are the solution, minimal solution, and maximal solution of the IVP $\left(E_{3}\right)$ and that $x=\psi(t), \psi_{m}(t)$, and $\psi_{M}(t)$ are the solution, minimal solution, and maximal solution of the IVP $\left(E_{4}\right)$, respectively. Then

$$
\phi(t) \leq \psi_{M}(t) \quad \text { and } \quad \phi_{m}(t) \leq \psi(t)
$$

for $t \in[0, T]$. 
Proof Consider the initial value problem

$$
\left(E_{\epsilon}\right): \quad{ }^{c} D^{p} x=F(t, x)+\epsilon, \quad x^{(k)}(0)=x_{k}, k=0,1,2, \ldots, n-1,
$$

where $\epsilon>0$ is a small positive number. By Theorem 3 the $\operatorname{IVP}\left(E_{\epsilon}\right)$ has at least one solution. Denote it by $\psi(t, \epsilon)$. Since $f(t, x) \leq F(t, x)<F(t, x)+\epsilon, t \in[0, T]$, by Theorem 2 we have $\phi(t)<\psi(t, \epsilon)$ for $t \in(0, T]$. Since $\lim _{\epsilon \rightarrow 0} \psi(t, \epsilon)=\psi_{M}(t)$ uniformly on $0 \leq t \leq T$, we obtain

$$
\phi(t) \leq \psi_{M}(t), \quad t \in[0, T]
$$

Just replacing $\epsilon$ with $-\epsilon$, the other inequality is proved similarly. Thus, the proof is completed.

\section{Conclusion}

In this paper, we obtained comparison principles for fractional differential equations with the Caputo derivatives of order $p(0 \leq n-1<p \leq n)$ under strict and nonstrict inequalities. Then the local existence and extremal solutions of fractional differential equations involving the Caputo derivatives were proposed. The results presented in this paper may be applied for further study of the quantitative properties of fractional differential equations with the Caputo derivatives.

\section{Acknowledgements}

The authors are thankful to the reviewers for their useful corrections and suggestions, which improved the quality of this paper.

Funding

This work is supported by National Natural Science Foundation of China (Grant No. 61673011).

Competing interests

The authors declare that they have no competing interests.

\section{Authors' contributions}

Both authors contributed equally and significantly in writing this paper. Both authors read and approved the final manuscript.

\section{Publisher's Note}

Springer Nature remains neutral with regard to jurisdictional claims in published maps and institutional affiliations.

Received: 14 March 2018 Accepted: 19 May 2018 Published online: 11 July 2018

\section{References}

1. Oldham, K.B., Spanier, J.: The Fractional Calculus. Academic Press, New York (1974)

2. Podlubny, I.: Fractional Differential Equations. Academic Press, San Diego (1999)

3. Kilbas, A.A., Srivastava, H.M., Trujillo, J.J.: Theory and Application of Fractional Differential Equation. Elsevier, Amsterdam (2006)

4. Hadid, S.B., Ta'ani, A.A., Momani, S.M.: Some existence theorems on differential equations of generalized order through a fixed-point theorem. J. Fract. Calc. 9, 45-49 (1996)

5. Lakshmikantham, V., Vatsala, A.S.: Theory of fractional differential inequalities and applications. Commun. Appl. Anal. 11, 395-402 (2007)

6. Lakshmikantham, V.: Theory of fractional functional differential equations. Nonlinear Anal., Theory Methods Appl. 69 , 3337-3343 (2008)

7. Lakshmikantham, V., Vatsala, A.S.: Basic theory of fractional differential equations. Nonlinear Anal., Theory Methods Appl. 69, 2677-2682 (2008)

8. Denton, Z., Vatsala, A.S.: Fractional integral inequalities and applications. Comput. Math. Appl. 59, 1087-1094 (2010)

9. Denton, Z., Ramírez, J.D.: Existence of minimal and maximal solutions to RL fractional integro-differential initial value problem. Opusc. Math. 37, 705-724 (2017)

10. Yu, C., Gao, G.: Existence of fractional differential equations. J. Math. Anal. Appl. 310, 26-29 (2005)

11. Yakar, A.: Some generalizations of comparison results for fractional differential equations. Comput. Math. Appl. 62, 3215-3220 (2011) 
12. Vasundhara Devi, J., Mc Rae, F.A., Drici, Z.: Variational Lyapunov method for fractional differential equations. Comput. Math. Appl. 64, 2982-2989 (2012)

13. Ibrahim, R.W., Momani, S.: On the existence and uniqueness of solutions of a class of fractional differential equations. J. Math. Anal. Appl. 334, 1-10 (2007)

14. Deng, J., Ma, L.: Existence and uniqueness of solutions of initial value problems for nonlinear fractional differential equations. Appl. Math. Lett. 23, 676-680 (2010)

15. Wang, F: Existence and uniqueness of solutions for a nonlinear fractional differential equation. J. Appl. Math. Comput. 39, 53-67 (2012)

16. Kosmatov, N.: Integral equations and initial value problems for nonlinear differential equations of fractional order. Nonlinear Anal., Theory Methods Appl. 70, 2521-2529 (2009)

17. Hu, T., Qian, D., Li, C.: Comparison theorem of fractional differential equations. Commun. Appl. Math. Comput. Sci. 23, 97-103 (2009)

18. $\mathrm{Xu}, \mathrm{Y}:$ : Comparison principle and solution bound of fractional differential equations. In: Proceedings of the ASME IDET-CIE Conference Boston, Massachusetts, USA (2015)

19. Yu, H., Bao, S., Liu, X.: A new comparison theorem of fractional differential equations. Math. Pract. Theory 46, 1-8 (2016)

\section{Submit your manuscript to a SpringerOpen ${ }^{\odot}$ journal and benefit from:}

- Convenient online submission

- Rigorous peer review

- Open access: articles freely available online

- High visibility within the field

- Retaining the copyright to your article

Submit your next manuscript at $\boldsymbol{~ s p r i n g e r o p e n . c o m ~}$ 